\title{
Quartäre kalkige Äolianite von N-Menorca (Balearen, westliches Mittelmeer)
}

\author{
Dierk Henningsen *)
}

Eolianite, biocalcarenite, petrography, modal analysis, Pleistocene, foraminifers

Balearic Islands, Minorca

\begin{abstract}
Kurzfassung: Vom Norden der Insel Menorca (Balearen, westliches Mittelmeer) werden drei Serien von kalkigen Äolianiten beschrieben. In ihrer petrographischen Zusammensetzung unterscheiden sich Proben aus der älteren von solchen aus den beiden jüngeren Serien aufgrund des fast völligen Fehlens von silikatischen Mineralen und Gesteinsresten sowie - innerhalb der Biogene - durch ein Vorherrschen von Schalen von Foraminiferen gegenüber Resten von Kalkalgen. Vermutlich entstanden die Jüngsten Äolianite während des letzten Glazials, während die Jüngeren in das frühe Pleistozän und die Älteren an den Übergang Pliozän/ Pleistozän gestellt werden müssen. Damit wird deutlich, $\mathrm{da} ß$ es offenbar auf den Balearen nicht regelmäßig in jedem Glazial zur Bildung von Äolianiten gekommen ist.
\end{abstract}

[Quaternary calcareous Eolianites from Northern Minorca (Balearic Islands, Western Mediterranean)]

\begin{abstract}
Three different series of calcareous eolianites are described from Northern Minorca (Balearic Islands, Western Mediterranean). The petrographic composition of samples from the three series is not uniform: in the oldest series, contrary to the younger ones, only very few siliceous minerals or rock fragments are present, among the bioclasts there are more tests of forams than fragments of calcareous algae. Presumably the youngest eolianites originated during the last glacial period, the older eolianites during the early Pleistocene and the oldest near the transition of the Pliocene to the Pleistocene. This indicates that most likely in the Balearic region the formation of eolianites did not take place regularly during every glacial period.
\end{abstract}

\section{Einleitung}

Die im Bereich des westlichen Mittelmeeres vor allem an den Inselküsten verbreiteten kalkigen Äolianite werden allgemein als Bildungen von aridem Klima

*) Anschrift des Verfassers: Prof. Dr. D. Henningsen, Institut für Geologie und Paläontologie der Universität Hannover, Callinstr. 30, D - 3000 Hannover 1. und Regressionsphasen, also Glazialzeiten, angesehen. Bei abgesenktem Meeresspiegel wurden aus den trockengefallenen Schelfgebieten die nicht viel vorher abgelagerten Fossilreste und andere sandige Komponenten auf das Land geweht. Wo die Äolianite sich in verschiedene Serien gliedern lassen - wie z. B. auf den Balearen - ist die Frage wichtig, ob und wieweit sich die verschiedenen Serien mit bestimmten pleistozänen Kaltzeiten (Glazialen) parallelisieren lassen. Hierbei gibt es trotz einiger Bearbeitungen durchaus noch erhebliche Probleme, wie im folgenden an dem Beispiel eines Teilbereiches (Halbinsel Cavalleria bzw. Caballeria, Bucht von Tirant, s. Abb. 1) aus dem $\mathrm{N}$ der Insel Menorca gezeigt werden soll.

Die auf den Balearen oft als „marés” bezeichneten kalkigen Äolianite sind überwiegend windabgelagert, zeigen aber vielfach auch Zeichen einer Sedimentation in (z. B. im früheren Strandbereich) oder Umlagerung durch fließendes Wasser (z. B. in Verbindung mit Abschwemmvorgängen). In Menorca überlagern die Äolianite diskordant Gesteine des Tertiärs, Mesozoikums oder Jungpaläozoikums. Im N-Teil der Insel wird seit längerem eine ältere von einer jüngeren Serie abgetrennt: Die ältere kommt vor allem auf der Halbinsel Cavalleria und östlich von Fornells vor; sie wurde von BOURROUILH \& MAGNÉ (1963) und BOURROUILH (1973) aufgrund von Funden von Foraminiferen und Landschnecken an den Übergang Pliozän/Pleistozän gestellt, während sie von MERCADAL et al. (1970) hauptsächlich wegen ihrer Landschnecken-Fauna als vermutlich Mindel-zeitlich eingestuft wurde. CUERDA (1975) nennt in seiner zusammenfassenden Übersicht beide Auffassungen, ohne sich einer von beiden anzuschließen.

Die jüngeren Äolianite an der Bucht von Tirant (Cala Tirant) wurden von BOURROUILH (1973: Abb. 11 und Anl. 3) zwar nach ihrer Höhenlage (unterhalb bzw. 


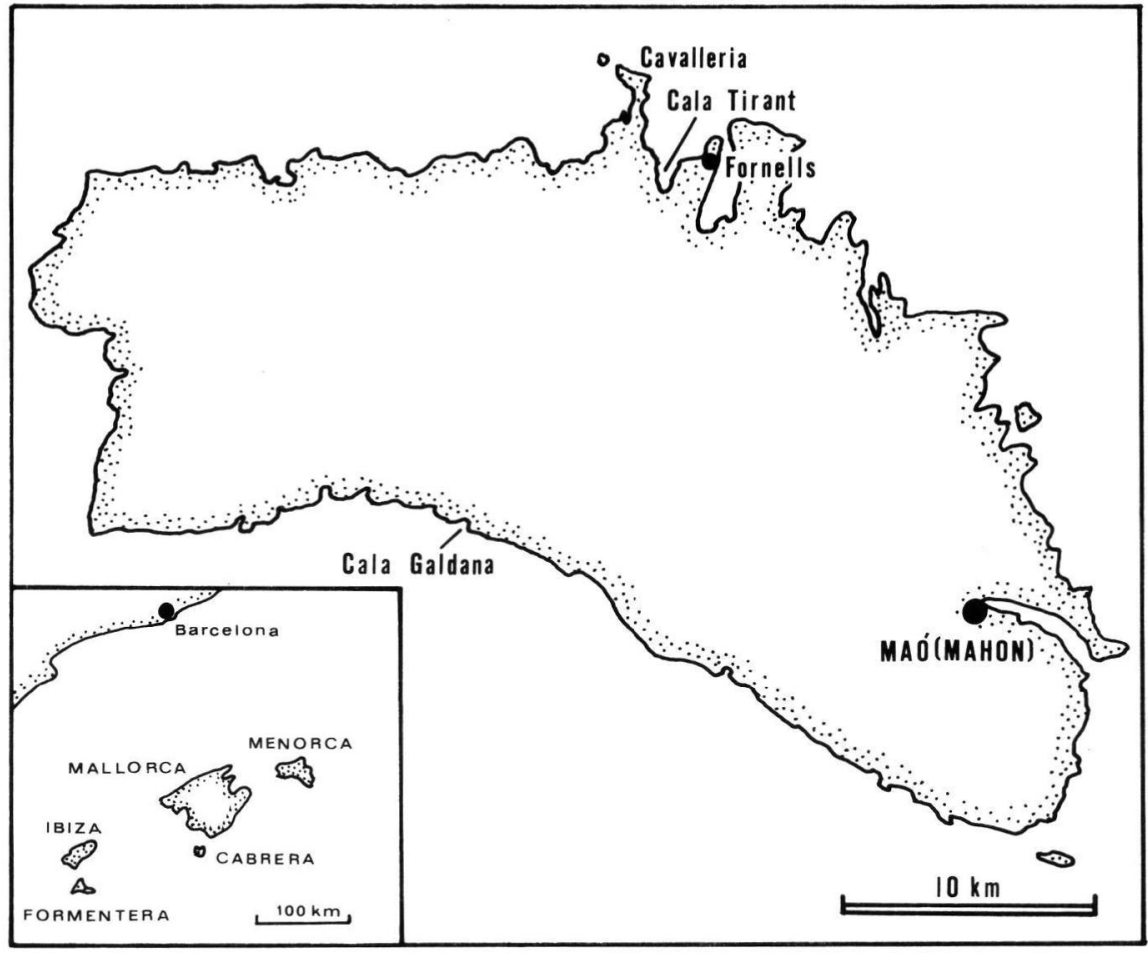

Abb. 1:

Übersichtskarte der Insel Menorca mit Lage der im Text erwähnten Lokalitäten.

oberhalb von $40 \mathrm{~m}$ üb. NN) kartiert, aber sonst nicht weiter unterschieden. Auch Mercadal et al. (1970) nennen von der Cala Tirant nur Äolianite der WürmZeit, die sie zwischen den Meereshochständen des Paläotyrrhens und Eutyrrhens einordnen. Von anderen Lokalitäten Menorcas listen sie aber auch ältere Äolianite auf, die sie dem Riß-Glazial zuweisen. Diese Einstufung wird ebenfalls mit dem Auftreten bzw. Nichtvorhandensein von Gehäusen bestimmter Landschnecken begründet. Sie paßt sich einem Altersschema an, wie es von BUTZER \& CUERDA (1962) und CUERDA (1975) für die Äolianite der Insel Mallorca entwickelt worden war, wobei auch unterschiedliche Höhenlagen von Strandterrassen einbezogen wurden, ohne daß auf den Einfluß von auf den Balearen sehr differenzierten jungen Vertikalbewegungen ausreichend eingegangen worden wäre.

Wenig ist bisher über die petrographische Zusammensetzung der Äolianite Menorcas und möglichen Unterschiede entsprechend ihrem Bildungsalter bekannt. BOURROUILH (1973) machte einige Angaben vor allem über die Älteren Äolianite. Eine Untersuchung von Proben aus verschiedenen alten Serien wie auf der Nachbarinsel Mallorca, wo CALVET etal. (1980) feststellten, daß mit zunehmendem Alter die weniger stabilen Komponenten Aragonit und $\mathrm{Mg}$ reicher Kalzit abnehmen - wobei allerdings große Streuungen auftreten - liegt von Menorca nicht vor.
Ziel dieser Arbeit ist es, die im Gelände gewonnenen Hinweise für eine Untergliederung der Äolianite in N-Menorca mit Untersuchungen ihres Stoffbestandes $\mathrm{zu}$ verbinden und anschließend ihre jeweilige Altersstellung zu erörtern. In einigen Punkten wurden dabei Arbeitsergebnisse von H. SCHMIDT (1989) berücksichtigt; das hier vorgelegte Konzept hat aber allein der Verfasser zu vertreten.

Die Geländearbeiten für diese Untersuchung erfolgten in Absprache mit der Comisión de Geología in Madrid (Vorhaben 7/87). Sie wurden hauptsächlich im Sommer 1987 durchgeführt, wobei dankenswerterweise die Hannoversche Hochschulgemeinschaft einen Reisekosten-Zuschuß an H. SCHMIDT und den Verfasser gewährte.

\section{Geländebeobachtungen}

Die $\ddot{A} l$ testen $\ddot{A}$ olia nite im N von Menorca haben meist eine weiße oder rötliche Farbe. Sie sind etwa $50 \mathrm{~m}$ mächtig. Das in der Regel fest zementierte kalkige Gestein ist häufig laminiert und weist Schrägschichtungslamellen auf, die mit meist geringen Winkeln (weniger als $30^{\circ}$ ) in wechselnde Richtungen einfallen. Auf der Halbinsel Cavalleria treten innerhalb der Schichtfolge Diskordanzen, teilweise mit Lagen von Kalkstein-Geröllen und Rotlehm- 

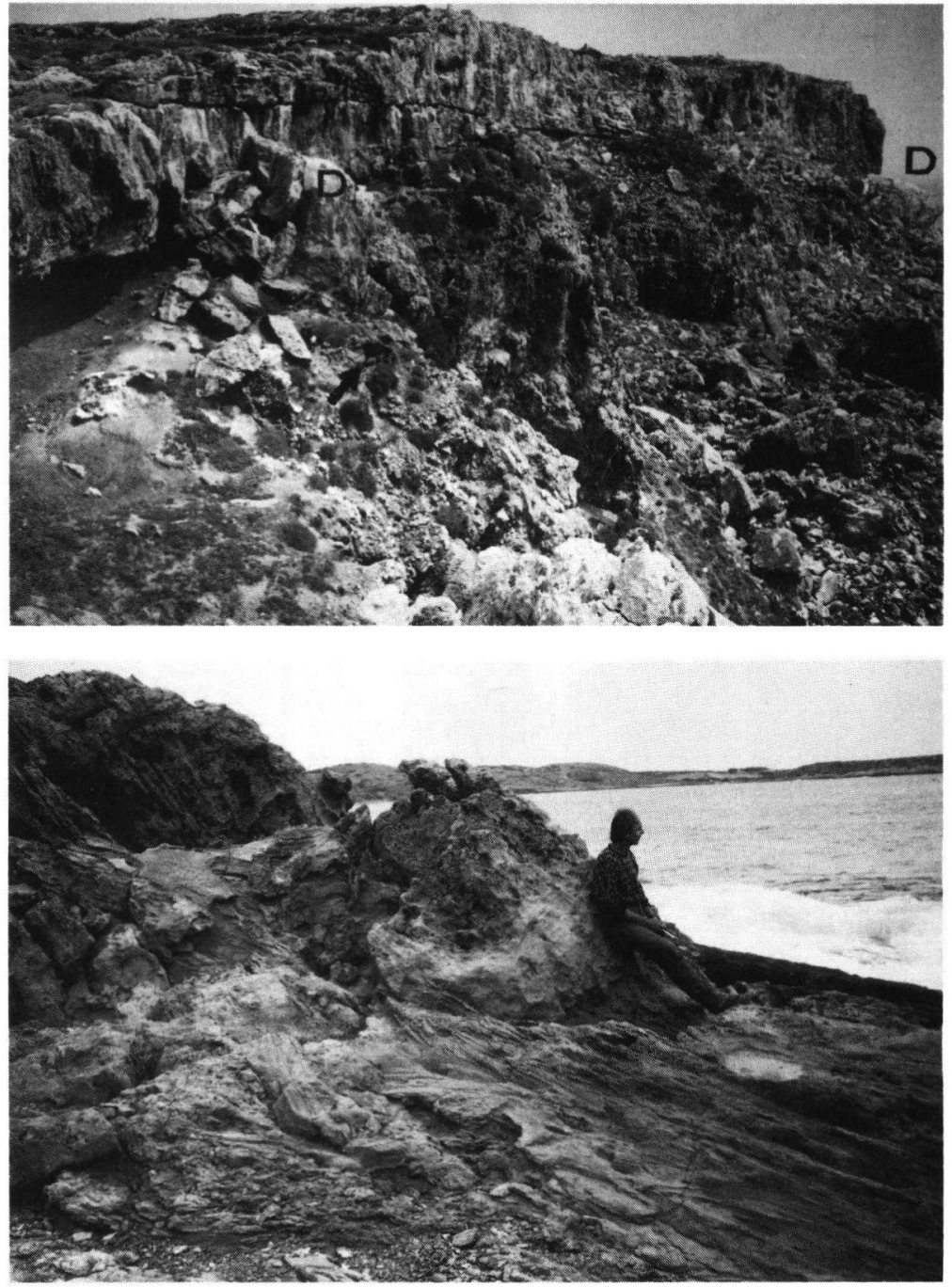

Abb. 2: Ältere Äolianite, etwa $10 \mathrm{~m}$ mächtig, über dolomitisierten Kalksteinen der Jura-Zeit. Diskordanzen (D) innerhalb der Äolianite und an ihrer Basis, teilweise mit Rotlehmen gefüllt. Pfeil in Bildmitte $=$ Ziege als Größenmaßstab. Halbinsel Cavalleria.
Abb. 3: Heruntergebrochenes Paket von Jüngeren Äolianiten mit steilem Einfallen, teilweise (rechte Bildseite) überlagert von flach meerwärts geneigten, hier geringmächtigen Jüngsten Äolianiten.

E-Seite Bucht von Tirant.
Bildungen, auf (Abb. 2). Lokal gibt es einzelne Schlottenfüllungen mit gelblichen Jüngeren oder Jüngsten Äolianiten. Die Unterseite der Ältesten Äolianite liegt etwa horizontal in einer Höhe von etwa $40 \mathrm{~m}$ üb. NN, biegt aber örtlich auf weniger als $20 \mathrm{~m}$ üb. NN herunter, vermutlich infolge junger tektonischer Verstellungen. Die Oberseite dieser Serie erreicht in der Nähe des Leuchtturms an der Nordseite der Halbinsel Cavalleria eine Höhenlage von etwa $90 \mathrm{~m}$ üb. NN.

Trotz des Auftretens von Wurzelröhren, Gehäusen von Landschnecken und dem Pilz-artigen Rest Microcodium sowie speziellen Schrägschichtungsformen, die von MERCADAL et al. (1970) als Hinweise auf eine festländisch-äolische Bildung angesehen wurden, macht das Gestein im Sinne von BOURROUILH \& MAGNÉ (1963) und BourrouilH (1973) eher den
Eindruck einer Bildung eines flachmarinen Bereichs, der allerdings häufig trockengefallen ist.

Der jüngere Marés an der E-Seite der Cala Tirant läßt sich in Erweiterung der bisherigen Angaben in der Literatur in zwei Serien unterteilen, die im folgenden als Jüngere und Jüngste Äolianite bezeichnet werden.

Die etwa 5-10 m mächtigen J ü n g e r e n $\ddot{A}$ o $\mathrm{l}$ i a $\mathrm{n}$ i t e haben überwiegend eine graue Farbe. Sie sind meist gut zementiert und relativ gleichkörnig ausgebildet (überwiegend Mittelsand-Bereich). Schutt- und Geröll-Lagen kommen gelegentlich vor. Vorhandene Schrägschichtungslamellen fallen mehrfach steiler als $30^{\circ}$, meistens in westlicher Richtung, ein. Die Jüngeren Äolianite sind von der Küstenlinie bis auf Höhen von mehr als $60 \mathrm{~m}$ üb. NN zu verfolgen, wobei auffällt, daß ihre Unterseite nicht immer 
mit dem heutigen Relief übereinstimmt: An der ESeite der Cala Tirant steigt z. B. die Basis der Jüngeren Äolianite in NNE-Richtung hoch, obwohl hier ein Tal eingeschnitten ist, das offensichtlich während der Ablagerung der Serie in dieser Form noch nicht vorhanden war.

Eine Besonderheit zeigt ein am N-Rand der Ortschaft Tirant, ca. $1 \mathrm{~km} \mathrm{~N}$ des Südendes der Bucht gelegener Aufschluß an der Küste: Eine Scholle der Jüngeren Äolianite ist infolge der Einwirkung einer früheren Brandung herabgebrochen und liegt heute mit steilem Einfallen unmittelbar auf der Strandplattform aus devonischen Sand- und Tonsteinen. Er wird teilweise bedeckt von flachlagernden Jüngsten Äolianiten (Abb. 3).

Die J üngsten Ä o li a n i t e sind ebenfalls etwa bis zu 5-10 m mächtig; sie haben eine gelbe bis gelblich-bräunliche Farbe. Sie kommen vor allem an der E-Seite der Cala Tirant und deren Fortsetzung in Richtung Fornells vor. Das mittel- bis grobsandige Material ist teilweise fest kalkig zementiert, teilweise aber auch mürbe. Die Jüngsten Äolianite in dem untersuchten Bereich enthalten zahlreiche Schutt-und Geröll-Zwischenlagen (Material: devonische Sand-, Ton- und Mergelsteine von benachbarten Felsen und Bergrücken; Geröll/Fragment-Durchmesser bis mehr als $50 \mathrm{~cm}$ ), die oft in meerwärtiger Richtung zunehmen. Sie weisen ebenso wie bis mehr als $1 \mathrm{~m}$ Durchmesser erreichende Rinnenfüllungen auf Transport und Umlagerung durch Wasser hin, daneben kommen aber auch Anzeichen für äolische Sedimentation vor, wie steil einfallende Schrägschichtung oder horizontal liegende planare Lamination. Aus allem ergibt sich ein vorwiegend äolischer Antransport mit festländisch-aquatischer Umlagerung.

Die Jüngsten Äolianite reichen sicherlich bis unter den heutigen Meeresspiegel herunter, auf dem Land ziehen sie sich in Anlehnung an das heutige Relief bis in Höhen von etwa $60 \mathrm{~m}$ üb. NN hinauf. Im Bereich der E-Seite der Cala Tirant zeigen die Schrägschichtungslamellen eine Anwehung aus nördlicher bis nordöstlicher Richtung an. Dieses hatte auch BouRROUILH (1973: 500) beobachtet, ebenso wie die vorher bei den Jüngeren Äolianiten beschriebene Herkunft aus westlicher Richtung. Da er die Äolianite aber als eine Einheit ansah, mußte er zum Ergebnis kommen, daß eine Ablagerung allein durch Wind bei derart verschiedenen Transportrichtungen unwahrscheinlich sei.

\section{Zusammensetzung der Äolianite}

In Gesteinsdünnschliffen von Äolianit-Proben aus den drei beschriebenen Serien wurde deren Zusammensetzung ermittelt. Im Prinzip treten dieselben Komponenten auf, wie sie ausführlich von gleichartigen Ablagerungen auf den Balearen-Inseln Ibiza und Formentera beschrieben wurden (HENNINGSEN, KELLETAT \& HAGN 1981) und in ähnlicher Ausbildung u. a. auch von der Kanaren-Insel Fuerteventura bekannt sind (z. B. ROTHE 1986). Im folgenden wird auf eine Einzelbeschreibung der Komponenten verzichtet. Diese wurden nach der Auszählung zu folgenden Gruppen zusammengefaßt (Tab. 1):

1. Silikatische Minerale und Lithoklasten (Einzelkörner von Quarz, seltener Feldspäte, Glimmer und Schwerminerale; Fragmente/Gerölle von Sandsteinen, Quarziten, Silt- und Tonsteinen sowie Kieselgesteinen)

2. Karbonatische Lithoklasten und Abiogene (Fragmente von Kalksteinen, Intraklasten, Peloide, Koprolithen u.a.)

3. Biogene (hauptsächlich Reste/Schalen von Rotalgen, Foraminiferen, außerdem von Bryozoen, Crustaceen, Echinodermen, Serpuliden u. a.)

4. Grundmasse/Zement (mikritischer oder sparitischer Zement, teilweise mit geringen Anreicherungen von Fe-Hydroxiden; Porenfüllungen).

Tab. 1: Zusammensetzung von Äolianit-Proben aus N-Menorca:

Arithmetische Mittelwerte in Vol. \% mit Standard-Abweichung (s). Zählergebnisse nach H. SCHMIDT (1989), ergänzt.

Ältere

Jüngere

Jüngste Äolianite

$\begin{array}{rr}1 & 14 \\ 9 & 9 \\ 9 & 25 \\ 8 & 38 \\ 8 & 14\end{array}$

7
3
12
9
7

$\begin{array}{rr}7 & 6 \\ 8 & 3 \\ 40 & 6 \\ 26 & 12 \\ 19 & 10\end{array}$


Bei der Ansprache bzw. Auszählung war es manchmal schwierig, zwischen wenig strukturierten Biogenen und karbonatischen Lithoklasten zu unterscheiden. Außerdem ist zu beachten, daß der Gesamt-Porenraum eher etwas höher als die in Tab. 1 angegebenen Werte ist, weil kleine Hohlräume in Biogenen (z. B. leere Kammern von Foraminiferen) nicht berücksichtigt wurden.

Ausgezählt wurden insgesamt 25 Proben ( 7 aus den Älteren und je 9 aus den Jüngeren und Jüngsten Äolianiten). Alle haben eine Korngrößen-Zusammensetzung im Mittelsand-Bereich, sind also insofern direkt miteinander vergleichbar. Gezählt wurden pro Probe zwischen 200 und 500, in der Mehrzahl der Fälle zwischen 300 und 400 Punkte.

Als Ergebnis der Auszählung ist festzuhalten, daß die Gehalte an einzelnen Komponenten, insbesondere die von Grundmasse/Zement und die der Porenräume, von Probe zu Probe stark schwanken. Proben aus der älteren Serie unterscheiden sich deutlich von denen der beiden jüngeren Serien, während die Proben aus diesen beiden im Mittel relativ gleichartig ausgebildet sind (Tab. 1). Im einzelnen gelten folgende typische Merkmale:

Äl t e r e S e r i e : Keine oder fast keine silikatischen Komponenten/Lithoklasten, unter den Biogenen vorherrschend Foraminiferenschalen

Jüngere und jüngste Serie : Deutlicher Gehalt an silikatischen Komponenten, dieser meist bei den Jüngeren Äolianiten höher. Das ist angesichts eines vermutlichen Antransportes aus westlicher Richtung verständlich, weil im W der Bucht von Tirant paläozoische Sand- und Kieselgesteine eine größere Verbreitung haben als in N- und NE-Richtung (Herkunftsrichtung der jüngsten Serie).

Unter den Biogenen herrschen Rotalgen-Reste (Corallinaceen) weitaus vor, wobei insgesamt in Proben aus der jüngsten Serie der Gehalt an Biogenen höher ist, während in denen aus der jüngeren Serie reichlicher Grundmasse/Zement vorkommt. Beim Porenraum bestehen keine generellen Unterschiede zwischen den verschiedenalten Proben. Hierbei sind wie in Ibiza (Henningsen, Kelletat \& Hagn 1981: 115) lokale Verhältnisse (z. B. Nähe zum Meer) offenbar wichtiger als eine mit höherem Alter zu erwartende Zunahme der sekundären Porenfüllungen.

\section{Altersstellung der Äolianit-Serien}

Die Äolianite der Jüngsten Serie auf N-Menorca sollten ebenso wie die jüngsten Äolianite auf den Nachbarinseln Mallorca und Ibiza dem Würm- bzw.
Weichsel-Glazial zugeordnet werden. In Jüngsten Äolianiten von der S-Küste Menorcas (Cala Galdana) tritt z. B. die Foraminiferen-Gattung Amphisorus hemprichii auf, die im Mittelmeer-Raum als Hinweis auf das Jungpleistozän angesehen wird (Frdl. Mitteilung von Prof. Dr. H. HAGN/München).

Die Jüngeren Äolianite von N-Menorca repräsentieren sicherlich eine Kaltzeit vor dem Würm-/WeichselGlazial. Ob diese aber im Sinne einer „Abzählstratigraphie" dem Riß-/Saale-Glazial entspricht, ist zweifelhaft.

Bemerkenswert ist eine Übereinstimmung mit den Verhältnissen auf Mallorca insofern, als dort ebenfalls während der Ablagerung der jüngsten Serie die Winde hauptsächlich aus östlicher, während der Anwehung der nächst älteren Serie aber aus westlicher Richtung kamen (BUTZER \& CUERDA 1962: 413). Ein Vergleich zwischen Menorca und Ibiza/Formentera zeigt Übereinstimmungen darin, daß dort zwischen den Äolianiten der jüngsten und denen der älteren Serie keine auffälligen Unterschiede festzustellen sind. Die älteren Äolianite von Ibiza müssen aber nach HAGN (in HeNNINGSEN, KeLLETAT \& HAGN 1981: 121) aufgrund ihrer Foraminiferen-Fauna in das frühe Pleistozän (Emilianum) eingestuft werden. Ähnliche Äolianite von der S Mallorca gelegenen Insel Cabrera waren von MATEU, Colom \& CUERDA (1979) auch in das frühe Pleistozän gestellt worden. Die stratigraphische Zuordnung des Emilianums wird im Bereich des W Mittelmeeres nicht einheitlich vorgenommen. Wenn es, wie es zumeist geschieht, mit einer Kaltzeit parallelisiert wird, müßte es der Günz-/ Menap-Zeit oder einem anderen Glazial im frühen Pleistozän entsprechen (z. B. CUERDA 1975: 18; KRÖMMELBEIN/STRAUCH 1986: Tab. 21). Im Gegensatz zu BUTZER \& CUERDA ((1962) und CUERDA (1975) ist also festzuhalten, daß es von Mallorca keine eindeutigen Datierungen für Äolianite aus dem Rißoder Mindel-Glazial, dafür aber von der vorlagernden Insel solche für ein noch älteres Glazial gibt.

Die Älteren Äolianite N-Menorcas unterscheiden sich, wie oben beschrieben, sowohl in ihrer Zusammensetzung als auch durch ihre meist stärkere Zementation deutlich von den Jüngeren und Jüngsten Äolianiten. Vergleichbare Gesteine wurden vom Verfasser auf Ibiza nicht beobachtet; ob sie auf Mallorca vorkommen, wie CUERDA (1975) vermuten läßt, bleibt offen. Sie müssen in jedem Fall älter als die Serien aus dem Emilianum sein; eine Alterseinstufung am Übergang Pliozän/Pleistozän im Sinne von BOURROUILH \& MAGNÉ (1963) und Bourroullh (1973) ist sehr wahrscheinlich.

In jedem Fall wird deutlich, daß es auf Menorca und den benachbarten Balearen-Inseln wahrscheinlich 
nicht in jedem Glazial regelmäßig zur Ausbildung einer Äolianit-Serie gekommen ist, sondern diese nur unter speziellen Bedingungen in wenigen Kaltzeiten des Quartärs stattfand. Nach Auffassung des Verfassers sind derartige Sonderbedingungen bisher erst nachgewiesen vom Übergang Pliozän/Pleistozän, aus dem frühen Pleistozän und aus dem Würm-I Weichsel-Glazial.

\section{Schriftenverzeichnis}

BOURROUilH, R. (1973): Stratigraphie, sédimentologie et tectonique de l'île de Minorque et du NE de Majorque (Baleares). - Thèse, Trav. Dép. Géol. Struct. Univ. Paris VI, 822 S.; Paris.

— \& MAGNÉ, J . (1963): A propos de dépôts du Pliocène supérieur et du Quarternaire sur la côte nord de l'île de Minorque (Baléares). - Bull. Soc. Géol. France, 7. sér., V: 298-302; Paris.

BUTZER, K. W. \& CUERDA, J. (1962): Coastal stratigraphy of southern Mallorca and its implications for the Pleistocene chronology of the Mediterranean Sea. - J. Geology, 70: 398-416; Chicago/III.

Calvet, F., Plana, F. \& Traveria, A. (1980). La tendencia mineralógica de las eoliniatas del Pleistoceno de Mallorca, mediante la aplicación del método de Chung. Acta Geol. Hispanica, XV, 2: 39-44; Barcelona.
CUERDA, J. (1975): Los tiempos Cuaternarios en Baleares. - 304 S., Palma de Mallorca (Diputación Provincial de Baleares).

Henningsen, D., Kelletat, D. \& Hagn, H. (1981): Die quartären Äolianite von Ibiza und Formentera (Balearen, Mittelmeer) und ihre Bedeutung für die Entwicklungsgeschichte der Inseln. - Eiszeitalter u. Gegenwart, 31: 109-133; Hannover.

KRÖMmelBein, K. \& StraUCH, F. (1986): BRINKMANNs Abriß der Geologie, 2. Bd.: Historische Geologie, 12./13. Aufl. - 404 S., Stuttgart (Enke).

Mateu, G., Colom, G. \& Cuerda, J. (1979): Los foraminiferos plio/pleistocenicos de la Isla de Cabrera (Baleares) y las condiciones paleoecologicas del antiguo Mar Balear. - Bol. Soc. Hist. Nat. Baleares, 23: 51-86; Palma de Mallorca.

Mercadal, B., Villalta, B., Obrador, J. F. \& Rosell, J. (1970): Nueva aportación al conocimiento del Cuarternario menorquín. - Acta Geol. Hispanica, V, 89-93; Barcelona.

Rothe, P. (1986): Kanarische Inseln. - Slg. Geol. Führer, 81: 226 S.; Berlin-Stuttgart (Borntraeger).

SCHмiDT, H. (1989): Marés von Menorca - quartäre äolische Karbonatsande und ihre Abgrenzung gegen flachmarine Bildungen. - Dipl.-Arbeit Univ. Hannover, 134 S.; Hannover. [Unveröff.]

Manuskript eingegangen am 25. 8. 1989. 